TORRES, J.L.R.; FABIAN,A.J.; POCAY, V.G. Níveis de adubação nitrogenada nas características morfológicas e produtividade do jiló. Horticultura Brasileira, Brasília, v. 21, n. 2, p. 166-169, abril/junho 2003.

\title{
Níveis de adubação nitrogenada nas características morfológicas e produtividade do jiló
}

\author{
José Luiz R. Torres ${ }^{1}$; Adelar José Fabian ${ }^{1}$; Vinícius G. Pocay ${ }^{2}$ \\ ${ }^{1}$ Centro Federal de Educação Tecnológica de Uberaba. Av. Edilson Lamartine Mendes, 300, 38000-045 Uberaba-MG; E-mail: \\ lrtorres@zaz.com.br; ${ }^{2}$ UNESP, Rod. Paulo Donato Castellane, s/n. Jaboticabal-SP
}

\section{RESUMO}

O experimento foi conduzido na UFRRJ, de julho a dezembro de 1993. Avaliaram-se os efeitos de diferentes níveis de nitrogênio $\left(0 ; 24 ; 48 ; 72\right.$ e $96 \mathrm{~kg} \mathrm{ha}^{-1}$ de $\left.\mathrm{N}\right)$ em algumas características morfológicas do fruto e na produtividade da cultura do jiló, cultivar Tinguá. As colheitas tiveram início a partir dos 105 dias após o plantio. Foram observados o diâmetro, comprimento, peso médio e produção total dos frutos nas colheitas realizadas num intervalo de dez dias, totalizando nove colheitas. Observou-se que o jiló responde positivamente à adubação nitrogenada aumentando sua produtividade em até $35,7 \%$, quando comparado à testemunha, apesar de não ter ocorrido diferença estatística entre as doses testadas. As características morfológicas avaliadas não foram influenciadas pelo nível de N.

\begin{abstract}
Levels of nitrogen fertilizer, morphologic characteristics of fruits and yield of jiló

The experiment was conducted at the Rural Federal University of Rio de Janeiro, Brazil, fron July to December, 1993. The effects of different levels of nitrogen $\left(0 ; 24 ; 48 ; 72\right.$ and 96 kg.ha- ${ }^{-1}$ of $\left.N\right)$ was evaluated over some morphological characteristics of the fruit and over yield of the jiló, cultivar Tinguá. Harvest began 105 days after sowing date, in 10-day intervals. The diameter, length, medium weight and total yield of fruits were evaluated. The jiló plant responds positively to the nitrogen fertilizer application, increasing the yield up to $35,7 \%$, in relation to the control plot, in spite of non statistical differences among nitrogen levels; the morphologic characteristics suffered no influence from $\mathrm{N}$ levels.
\end{abstract}

Keywords: Solanum gilo, nutrition.

Palavras-chave: Solanum gilo, nutrição.

(Recebido para publicação em 04 de maio de 2001 e aceito em 19 de março de 2003)

$\mathrm{O}$ jiló (Solanun gilo Raddi) é uma hortaliça tipicamente tropical, muito exigente em calor e pouco tolerante ao frio, podendo ser plantada o ano todo em localidades de inverno suave, como ocorre na Região Sudeste (PESAGRO/ RJ, 1989). Foram comercializados na CEASA-RJ 14.169,6 t no período de 1994 a 1997 provenientes dos Estados desta região, sendo 1,3 t de São Paulo, 304,6 t de Minas Gerais, 174,6 t do Espirito Santo e 13.689,1 t do Rio de Janeiro (95,1\% do total). No Estado do Rio de Janeiro, nas condições da Baixada Fluminense, importante região produtora, a planta forma um arbusto ramificado, apresentando frutificação com cachos de 2 a 4 frutos, com formas que variam de acordo com a cultivar, tem sabor amargo, sendo comercializados quando verdes medindo em média $4 \mathrm{~cm}$ de diâmetro por $6 \mathrm{~cm}$ de comprimento, com peso médio dos frutos entre 40 a $50 \mathrm{~g}$, podendo ser plantada o ano todo (PESAGRO/RJ, 2001). Morgado \& Dias (1992) avaliaram 43 genótipos de jiló e um de Solanum aethiopicum para caracterizar a coleção de germosplama da Embrapa Hortaliças e dentre outras, observaram grande variabilidade dos frutos, principalmente em relação à coloração e forma. A altura das plantas variou de 23 a $71 \mathrm{~cm}$, o peso unitário dos frutos variou de 19 a $110 \mathrm{~g}$ com o comprimento oscilando de 2,5 a $6,4 \mathrm{~cm}$. Quanto à coloração, 36\% dos genótipos apresentaram frutos verde escuros, $36 \%$ verde claros e quanto ao formato do fruto, $61 \%$ foram redondos, $20 \%$ achatados, $9,5 \%$ alongados e $9,5 \%$ ovais. A colheita inicia-se aos 105 dias após o plantio, podendo o período produtivo prolongar-se por 3 meses ou mais, dependendo do manejo (Filgueira, 1982).

Devido às características de crescimento e produção, comparando a outras plantas da família, o jiló apresenta uma maior distribuição de $\mathrm{N}$ em toda a planta, sendo muito exigente neste elemento do início ao final da cultura, tanto na folha e caule, como nos frutos (Furlani et al., 1978; Haag et al., 1978 e 1979), porém poucos trabalhos são encontrados relacionando adubação e produtividade na cultura. De Polli et al. (1990) destacam que a produtividade média do jiló no estado do Rio de Janeiro oscila entre 20 e 30 t.ha $^{-1}$, aplicando-se adubo orgânico no plantio e $60 \mathrm{~kg} \cdot \mathrm{ha}^{-1}$ de $\mathrm{N}$ em cobertura, (metade no transplantio e o restante 30 dias após). A produção média do jiló no período de 1994 a 1997 no estado do Rio de Janeiro ficou em torno de 29,1 t.ha-1 (PESAGRO, 2001).

Filgueira (2000) destaca que as cultivares Tinguá, Comprida Grande Rio e Morro Grande têm produtividade variável de 30 a 70 t.ha ${ }^{-1}$, utilizando 6 a 12 g.cova ${ }^{-1}$ de N (48 a 72 kg. ha ${ }^{-1}$, respectivamente). Casali et al. (1970) reportaram que para cultivares claras e compridas, obtiveram produtividades de 43,8 a 68,6 t.ha ${ }^{-1}$, com 21 a $27 \mathrm{~g}$ de peso médio dos frutos, e para cultivares es-

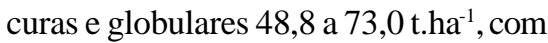
27 a $32 \mathrm{~g}$ de peso médio dos frutos.

Em outras solanáceas como o tomate, Pinto et al. (1997) observaram respostas significativas à adubação nitrogenada com aumento de produtividade de até $308 \%$, em relação ao tratamento sem nitrogênio, com aplicação de $90 \mathrm{~kg} \cdot \mathrm{ha}^{-1}$ de N, (1/3 no plantio e o restante 30 dias após); Silva et al. (2001) observaram que as maiores produções foram obtidas com plantas podadas e adensadas (149,2 t.ha $\left.{ }^{-1}\right)$ utilizando uréia 
como fonte, e as menores produções com plantas podadas $\left(91\right.$ t.ha $\left.^{-1}\right)$ e plantas não podadas $\left(63,2\right.$ t.ha $\left.{ }^{-1}\right)$ usando sulfato de amônia como fonte de N. Com pimentão, Valenzuela \& Romero (1996) observaram diminuição do número, peso e rendimento dos frutos com a dose de $180 \mathrm{~kg} \cdot \mathrm{ha}^{-1}$ de $\mathrm{N}$ aplicado no solo; Silva et al. (2001) não observaram alteração na produtividade da cultura do pimentão utilizando 3 doses de $\mathrm{N}$ aplicado ao solo $\left(13,3 ; 26,6\right.$ e 39,9 g.m $\left.{ }^{-2}\right)$, usando uréia como fonte. Com batata, Delazari et al. (1989) obtiveram respostas significativas nos tratamento com aplicação de nitrogênio (100; 200 e 300 kg.ha-1 de N) nas três unidades de solos estudados, porém, nos Latossolos Vermelho Amarelo Distróficos foram observados os maiores rendimentos econômicos da cultura.

Em outras culturas como rabanete, Cardoso \& Hiraki (2001) testando 3 doses de N $\left(100 ; 200\right.$ e 300 kg.ha $^{-1}$ de $\mathrm{N}$ ) e duas épocas de aplicação (9 e 20 dias após semeadura) observaram as maiores produções comerciais com a dose de $300 \mathrm{~kg} \cdot \mathrm{ha}^{-1}$ aplicado 9 dias após a semeadura. Com alho, Resende \& Souza (2001) testando 5 doses de N (40; $60 ; 80 ; 100$ e 120 kg.ha-1 de N) e três épocas de aplicação (30; 50 e 70 dias após o plantio), observaram que a maior produtividade ocorreu aplicando 40 t.ha ${ }^{1}$ de $\mathrm{N}$, independentemente da época, porém quanto mais tardia a aplicação menor a produtividade.

O presente trabalho teve o objetivo de avaliar o efeito de diferentes níveis de adubação nitrogenada em algumas características morfológicas dos frutos e na produtividade no jiló, em Seropédica, na safra 93/94.

\section{MATERIAL E MÉTODOS}

O trabalho foi desenvolvido no campo experimental da UFRRJ, de julho a dezembro de 1993, em uma área total de $500 \mathrm{~m}^{2}$. Mudas de jiló, cultivar Tinguá, verde clara comprida, foram produzidas em casa de vegetação, sendo transplantadas com 4 a 6 folhas, com 30 dias de idade para o local definitivo. O delineamento experimental foi de blocos ao acaso, com 5 tratamentos e 5 repetições, totalizando 25 parcelas. $\mathrm{O}$

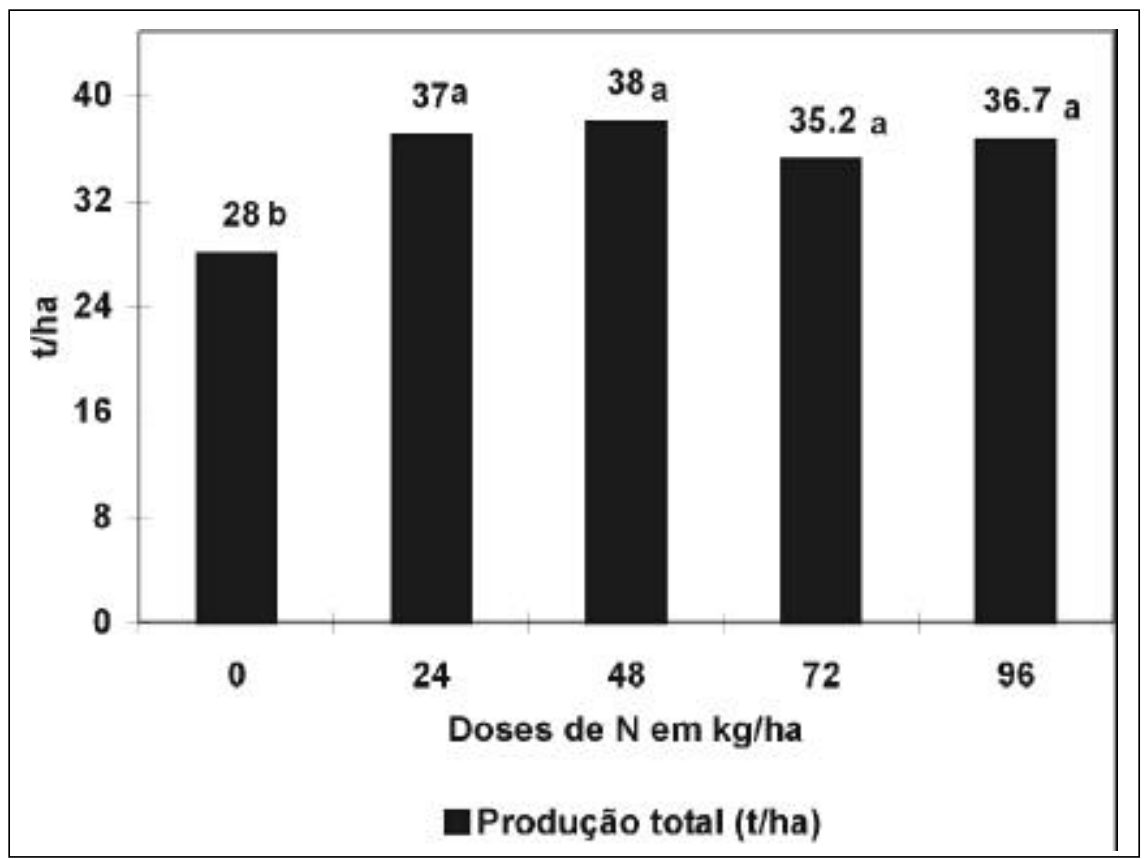

Figura 1. Produção total de jiló cultivado com cinco doses de nitrogênio, obtido em nove colheitas. Seropédica, UFRRJ, 1993.

experimento foi instalado com 16 plantas por parcela, no espaçamento de 1,25 $\mathrm{m} \times 1,00 \mathrm{~m}$, perfazendo um total de 20 $\mathrm{m}^{2}$ por parcela. O local teve o solo corrigido para os nutrientes que se fizeram necessários, com base na análise química do solo, de 0-20 cm: pH em $\mathrm{H}_{2} \mathrm{O}=$ 5,9; $\mathrm{Ca}=20 ; \mathrm{Mg}=7,5$ e $\mathrm{H}+\mathrm{Al}=18$ mmol $\mathrm{dm}^{-3} ; \mathrm{K}=32$ e $\mathrm{P}=30 \mathrm{mg} \cdot \mathrm{dm}^{-3}$; C. Org. $=0,7$ g.dm ${ }^{-3}$. De 20-40 cm: pH em $\mathrm{H}_{2} \mathrm{O}=5,8 ; \mathrm{Ca}=19 ; \mathrm{Mg}=4,0$ e $\mathrm{H}+$ $\mathrm{Al}=16 \mathrm{mmol} \mathrm{dm}^{-3} ; \mathrm{K}=48,4$ e $\mathrm{P}=30$ mg.dm ${ }^{-3}$; C. Org. $=0,8$ g.dm ${ }^{-3}$, seguindo as recomendações de adubação para a cultura e do estado do Rio de Janeiro (De Polli et al. 1990), sem aplicação de adubação orgânica, variando-se apenas as doses de nitrogênio.

$\mathrm{O}$ adubo nitrogenado foi parcelado em três vezes iguais: no transplantio das mudas para o campo, aos 15 e 45 dias após o transplantio, utilizando-se 5 doses $\left(0 ; 24 ; 48 ; 72\right.$ e 96 kg.ha $\left.{ }^{-1}\right)$ de N $(0$; 3; 6; 9 e 12 g.cova $\left.^{-1}\right)$ utilizando uréia como fonte, em cada aplicação. Aplicouse uma adubação básica para todos os tratamentos de $90 \mathrm{~kg} \cdot \mathrm{ha}^{-1}$ de $\mathrm{K}_{2} \mathrm{O}$ (na forma de $\mathrm{KCl}$ ) parcelado em duas aplicações, sendo metade no transplantio das mudas e o restante 45 dias após. Aplicou-se fósforo em dose única de 60 kg.ha ${ }^{-1}$ de $\mathrm{P}_{2} \mathrm{O}_{5}$ (32 g.cova ${ }^{-1}$ de $\left.\mathrm{P}\right)$ no transplantio, utilizando como fonte $\mathrm{O}$ superfosfato simples.
A colheita dos frutos teve inicio aos 105 dias após a semeadura em intervalos regulares de 10 dias, num total de nove colheitas no período do experimento. Após a colheita, os frutos foram medidos em seu diâmetro e comprimento com um paquímetro, pesados, obtendose o peso médio do fruto e o peso total em cada tratamento (Tabela 01), posteriormente transformados em t.ha ${ }^{-1}$.

Os dados climáticos utilizados foram coletados na Estação Meteorológica Experimental da PESAGRO-RJ/ Seropédica abrangendo todo o período produtivo da cultura. Os fatores determinados foram as temperaturas máxima, média e mínima e precipitação pluviométrica.

\section{RESULTADOS E DISCUSSÃO}

Todos os tratamentos supridos com nitrogênio apresentaram produtividade total superior ao tratamento testemunha, onde aplicações de 48; 24; 96 e 72 kg.ha- ${ }^{-1}$ de $\mathrm{N}$ resultaram em incremento de 35,7 ; $32 ; 31$ e $25,7 \%$, respectivamente (Figura 01), confirmando a constatação de que o jiló necessita de boa distribuição de $\mathrm{N}$ durante todo o seu ciclo e que normalmente responde positivamente à adubação nitrogenada com aumento na produtividade. 
Tabela 1. Diâmetro, comprimento, peso médio e produção total de frutos de jiló relacionados a diferentes doses de nitrogênio. Seropédica, UFRRJ, 1993.

\begin{tabular}{cccccc}
\hline \multirow{2}{*}{$\begin{array}{c}\text { Dose de } \mathbf{N} \\
\left(\mathbf{K g} \cdot \mathbf{h a}^{-1}\right)\end{array}$} & $\begin{array}{c}\text { Diâmetro Médio } \\
\text { do fruto } \mathbf{( c m )}\end{array}$ & $\begin{array}{c}\text { Comprimento } \\
\text { médio do fruto } \\
(\mathbf{c m})\end{array}$ & $\begin{array}{c}\text { Peso médio por } \\
\text { fruto } \mathbf{( g )}\end{array}$ & \multicolumn{2}{c}{ Produção total } \\
\hline 0 & $2,8 \mathrm{a}$ & $5,9 \mathrm{a}$ & $36,8 \mathrm{a}$ & $57,57 \mathrm{~b}$ & $28,0 \mathrm{~b}$ \\
24 & $2,7 \mathrm{a}$ & $5,9 \mathrm{a}$ & $36,9 \mathrm{a}$ & $73,84 \mathrm{a}$ & $37,0 \mathrm{a}$ \\
48 & $2,8 \mathrm{a}$ & $5,9 \mathrm{a}$ & $36,6 \mathrm{a}$ & $76,11 \mathrm{a}$ & $38,0 \mathrm{a}$ \\
72 & $2,8 \mathrm{a}$ & $5,8 \mathrm{a}$ & $36,9 \mathrm{a}$ & $70,42 \mathrm{a}$ & $35,2 \mathrm{a}$ \\
96 & $2,9 \mathrm{a}$ & $6,0 \mathrm{a}$ & $35,9 \mathrm{a}$ & $73,51 \mathrm{a}$ & $36,7 \mathrm{a}$ \\
\hline
\end{tabular}

Médias, dentro das colunas, seguidas de mesma letra não diferem entre si a 5\% de probabilidade pelo teste de Tukey (5\%).

$\mathrm{CV}(\%)=18,3$

Comparando-se a produtividade de frutos em cada colheita com as variações climáticas no período, constatouse a influência do clima sobre este fator, pois à medida que a temperatura média e a precipitação pluviométrica foram aumentando, proporcionalmente a produtividade também aumentou, atingindo os maiores valores nas três últimas colheitas $\left(7^{\mathrm{a}}, 8^{\mathrm{a}}\right.$ e $\left.9^{\mathrm{a}}\right)$ e em todas as doses de $\mathrm{N}$ avaliadas, que foram realizadas em dezembro: obteve-se com as doses de 24; 48; 72; 96 kg.ha ${ }^{-1}$ de $\mathrm{N}$ e testemunha: $10,7,16,7$ e 14,1; 9,0, 16,5 e 14,$2 ; 9,4,18,8$ e 12,$4 ; 11,8,15,1$ e 14,7 ; 8,$5 ; 15,3$ e 10,8 kg.ha ${ }^{-1}$ de frutos na $7^{\mathrm{a}}$, $8^{a}$ e $9^{\text {a }}$, respectivamente. No mês de dezembro ocorreu a maior precipitação pluviométrica (média 259,6 mm), temperatura máxima de 30,8 e mínima de $21,7^{\circ} \mathrm{C}$ e a média mais elevada $\left(25^{\circ} \mathrm{C}\right)$. Constatou-se assim que estes fatores climáticos influenciaram positivamente no desenvolvimento da cultura, o que está de acordo com a descrição da cultura feita pela PESAGRO/RJ (1989) que destaca que o jiló é uma planta exigente em calor e água, e a menor produção observada em julho e agosto, comprova que a planta é pouco tolerante ao frio e ao déficit hídrico, conforme destacado por Filgueira (2000).

As características morfológicas dos frutos têm grande importância para a comercialização pois estes devem estar dentro dos padrões exigidos pelo mercado consumidor. $\mathrm{O}$ fruto tem que ser colhido com a coloração verde clara brilhante, porém o diâmetro e o comprimento variam de acordo com a cultivar (Filgueira 2000). A cultivar apresentou diâmetro médio dos frutos variando en- tre 2,7 e $2,9 \mathrm{~cm}$ e comprimento entre 5,8 e $6,0 \mathrm{~cm}$ em todas colheitas realizadas, não sendo constatada diferença estatística entre os valores apresentados em todos os tratamentos (Tabela 01). Estes valores foram um pouco menores aos citados por Filgueira (1982; 2000) e pela PESAGRO-RJ (2001) que destaca valores médios de $4 \mathrm{~cm}$ para o estado do Rio de janeiro, porém nestas citações não são encontrados os intervalos entre colheitas, o que provavelmente pode justificar o tamanho dos frutos obtidos no experimento. Com relação ao comprimento do fruto também não houve diferença significativa entre os tratamentos, e o valor médio obtido ficou dentro dos padrões estabelecidos por Morgado \& Dias (1992) de 2,5 a 6,4 cm, para a cultura (Tabela 01). Filgueira (1982; 2000) e a PESAGRO (2001) destacam que o comprimento ideal para comercialização situa-se na faixa de 6 $\mathrm{cm}$, podendo-se deduzir que o fruto desenvolve mais rápido em termos de comprimento quando comparado ao diâmetro médio, atingindo seu tamanho ideal para comercialização, mesmo em intervalos de 10 dias.

Não houve influencia significativa dos tratamentos para o peso médio dos frutos. Esta é uma característica importante para comercialização do jiló, pois o mercado consumidor prefere frutos pesando entre 40 e 50 g cada (PESAGRO, 2001). Em todos os tratamentos avaliados no experimento obteve-se o peso médio do fruto variando de 35,9 a 36,9 g (Tabela 01), estando este peso bem acima dos valores destacados por Casali et al. (1970) que foi de 21 a 27 g. É possível que menor peso obtido pelos autores seja devido à cultivar avaliada, clara e comprida, de potencial genético inferior ao da cv. Tinguá. Por outro lado, os valores médios de peso do fruto citados pela PESAGRO-RJ (2001) estão um pouco acima dos valores encontrados neste experimento, possivelmente devido ao maior intervalo de colheita. Com relação ao trabalho de Morgado \& Dias (1992), os valores obtidos para peso médio do fruto no experimento ficaram dentro dos padrões estabelecidos, de 19 a $110 \mathrm{~g}$.

Os resultados observados evidenciaram o efeito significativo que as doses de nitrogênio tiveram sobre a produtividade dos frutos, apesar de não haver diferença estatística entre as 4 doses testadas $(24 ; 48 ; 72$ e 96 kg.ha-1 de N). Embora a menor produtividade obtida no experimento ter ocorrido no tratamento sem utilização de adubação nitrogenada (28 t.ha-1), este valor está dentro da faixa de produtividade destacada para a cultura no Estado por De Polli et al. (1990), de 20 a 30 t.ha $^{-1}$, utilizando $60 \mathrm{~kg} \cdot \mathrm{ha}^{-1}$ de $\mathrm{N}$, e próxima da produção média para o Rio de Janeiro (29,1 t.ha $\left.{ }^{-1}\right)$ citado pela PESAGRO-RJ (2001) usando a recomendação para o Estado. Cabe destacar que as condições climáticas ocorridas no período foram consideradas ideais para o desenvolvimento do jiló, cultura típica de clima quente. Não houve incidência de doenças na cv. avaliada, considerada rústica, resultante de programa de melhoramento genético da UFRRJ. Para confirmação dos dados obtidos, torna-se adequada a repetição do experimento por algumas safras. 


\section{J. L. R. Torres et al.}

Observou-se aumento da produtividade de jiló com a aplicação das doses de nitrogênio, porém este não foi acompanhado do aumento do diâmetro, comprimento e do peso médio dos frutos, no entanto ocorreu um maior número de frutos por planta, mais flores e frutos viáveis, mesmo com o intervalo de colheita aparentemente menor ao utilizado na descrição feita por Filgueira (1982; 2000) e PESAGRO-RJ (2001).

\section{LITERATURA CITADA}

CARDOSO, A.I.I.; HIRAKI, H. Avaliação de doses e épocas de aplicação de nitrato de cálcio em cobertura na cultura do rabanete. Horticultura Brasileira, Brasília, v. 19, n.3, p. 328 - 331, nov. 2001 CASALI, V.W.D.; CAMPOS, J.P.; COUTO F.A.A. Avaliação de introduções de jiló do Banco de Germoplasma de Hortaliças. In: CONGRES SO BRASILEIRO DE OLERICULTURA, 10, 1970, Viçosa-MG. Resumos....... Viçosa: SOB, 1970. p. $51-53$

DELAZARI, P.C.; ZANGRANDE, M.B.; FILHO N.D. Rendimento econômico da batata em função do nitrogênio, fósforo e potássio em solos do Espírito Santo. Horticultura Brasileira, Brasília, v. 7, n. 1, p. 24-27, 1989.

DE-POLLI, H.; ALMEIDA, D.L.; SANTOS, G.A.; CUNHA, L.H.; FREIRE, L.R.; SOBRINHO, N.M.B.A.; PEREIRA, N.N.C.; EIRA, P.A BLOISE, R.M.; SALEK, R.C. Manual de adubação para o Estado do Rio de Janeiro. Rio de Janeiro: UFRRJ, 1990. 179 p.
EMPRESA DE PESQUISA AGROPECUÁRIA DO ESTADO DO RIO DE JANEIRO. EMPRESA DE ASSISTÊNCIA TÉCNICA E EXTENSÃO RURAL DO ESTADO DO RIO DE JANEIRO. Recomendações para a cultura do jiló. Niterói: PESAGRO/1989.16 p. (Informe Técnico n. $\left.{ }^{\circ} 18\right)$.

EMPRESA DE PESQUISA AGROPECUÁRIA DO ESTADO DO RIO DE JANEIRO. A cultura do jiló: Perspectivas - Tecnologias - Viabilidade. Niterói: PESAGRO, 2001. 24 p. (PESAGRORIO. Documentos, 77).

FILGUEIRA, F.A.R. Manual de Olericultura: cultura e comercialização de Hortaliças v. 2. São Paulo: Agronômica Ceres, 1982. 357 p.

FILGUEIRA, F.A.R. Novo Manual de Olericultura: agrotecnologia moderna na produção e comercialização de hortaliças. Viçosa: UFV, 2000, $402 \mathrm{p}$.

FURLANI, A.M.C.; BATAGLIA, O.C.; FURLANI, P.R.; HIROCE, R.; GALLO, J.R.; BERNADI, J.B.; FORNASIERI, J.B. Composição mineral de diversas hortaliças. Bragantia, Campinas, v. 37. n. 5, p. 2-44, 1978.

HAAG, H.P.; MINAMI, K.; OLIVIERA, G.D.; SARRUGE, J.R. Nutrição mineral de hortaliças XXXII: distúrbios nutricionais em jiló (Solanun gilo cultivar Morro Grande oblongo) cultivado em solução nutritiva. Anais da ESALQ, v. 35, p. 327339, 1978.

HAAG, H.P.; OLIVEIRA, G.D.; MINAMI, K.; ROCHA FILHO, J.V.C. Nutrição mineral de hortaliças. XXXIII: acumulo de matéria seca e de nutrientes pela cultura do jiló (Solanun gilo cultivar Morro Grande oblongo). Anais da ESALQ, v. 36 , p. $77-88,1979$.
MORGADO, H.S.; DIAS, M.J.V. Caracterização da coleção de germosplama de jiló do CNPH/ EMBRAPA, Horticultura Brasileira, Brasília, v. 10, n. 2, p. $86-88,1992$.

PINTO, J.M.; SOARES, J.M.; COSTA, N.D.; CLEMENTINO, M.B.F.; BRITO, L.T.L.; SILVA, D.J. Dose e período de aplicação de nitrogênio via água de irrigação na cultura do tomate. Horticultura Brasileira, Brasília, v. 15, n. 1, p. 15 - 18, 1997.

RESENDE, G.M.; SOUZA, R.J. Doses e épocas de aplicação de nitrogênio sobre a produtividade e características comerciais de alho. Horticultura Brasileira, Brasília, v. 19, n. 2, p. 126-129, 2001. SILVA, M.A.G.; BOARETTO, A.E.; MURAOKA, T.; FERNANDES, H.G.; GRANJA, F.A.; SCIVITTARO, W.B. Efeito do nitrogênio e potássio na nutrição do pimentão cultivado em ambiente protegido. Revista Brasileira de Ciência do Solo, Campinas, v. 25, p. 913-922, 2001.

SILVA, E.C.; MIRANDA, J.R.P.; ALVARENGA, M.A.R. Concentração de nutrientes e produção de tomateiro podado e adensado em função do uso de fósforo, de gesso e de fontes de nitrogênio. Horticultura Brasileira, Brasília, v. 19, n. 1, p. 64-69, 2001.

VALENZUELA, J.L; ROMERO, L. Yield and optimum rate of nutrient in Capsicum annumm cv Lamuyo. Python Intern. J. Exp. Bot., v. 52, p. $63-75,1996$. 